patients. This 'clinical inertia' seems to apply across clinical care settings.

Minutolo et al. compared the management of cardiovascular risk factors in patients with type 2 diabetic nephropathy and an estimated glomerular filtration rate of $15-60 \mathrm{ml} / \mathrm{min} / 1.73 \mathrm{~m}^{2}$ who were followed in nephrology $(n=266)$, diabetology $(n=246)$ or primary care $(n=195)$ settings. In all three settings, low numbers of patients reached the target blood pressure level $(<130 / 80 \mathrm{mmHg})$, probably as a result of insufficient diuretic prescription and poor adherence to low-salt diets. More patients in the diabetology setting had total cholesterol in the target range $(63 \%)$ than did those in nephrology and primary care settings (59\% and $46 \%$, respectively). This was because statin prescription was greater in hypercholesterolemic patients in diabetology clinics than in those in nephrology and primary care settings $(70 \%, 50 \%$ and $41 \%$, respectively). Numbers of patients reaching the triglycerides target, however, were similar in all settings. Fewer patients in diabetology clinics than in nephrology and primary care settings attained the target glycated hemoglobin level of $<7 \%$ (32\%, 61\% and $46 \%$, respectively), despite more-frequent use of insulin plus oral hypoglycemic agents.

Patients with advanced diabetic nephropathy are, therefore, at risk of being under-treated, independently of the clinical setting. The authors suggest that a multidisciplinary approach might improve the management of these patients.

Original article Minutolo R et al. (2006) Management of cardiovascular risk factors in advanced type 2 diabetic nephropathy: a comparative analysis in nephrology, diabetology and primary care settings. J Hypertens 24: 1655-1661

\section{Exercise and an anabolic steroid improve muscle mass and function in hemodialysis patients}

Muscle wasting and weakness are common in hemodialysis patients, interfering with their ability to perform everyday activities and thereby reducing quality of life. A new study shows that nandrolone decanoate - a synthetic testosterone derivative-and resistance training increase muscle size and strength, and improve physical function, in maintenance hemodialysis patients.

Seventy-nine patients were randomized to receive either nandrolone (100 mg or $200 \mathrm{mg}$ once a week for women and men, respectively) or placebo injections, with or without lower extremity resistance exercise training during thrice-weekly dialysis sessions, for 12 weeks. Analysis of data from the 68 patients who completed the study showed that nandrolone, but not exercise alone, significantly increased lean body mass (average increase $3.1 \pm 2.2 \mathrm{~kg} ; P<0.001)$. Lower extremity exercise and nandrolone both markedly increased quadricep cross-sectional area $(P=0.02$ and $P<0.001$, respectively) in an additive manner. Muscles that were exercised became stronger $(P<0.0001$ for knee extension, hip abduction and hip flexion). Compared with their nonexercising counterparts, patients in the exercise groups reported improved physical functioning and less fatigue.

One participant withdrew from the nandrolone group because of interference with sexual function. The steroid also increased anger, an effect that was offset by exercise. The authors conclude that nandrolone and resistance training are safe and effective approaches to improving muscle weakness in stable hemodialysis patients.

Original article Johansen KL et al. (2006) Effects of resistance exercise training and nandrolone decanoate on body composition and muscle function among patients who receive hemodialysis: a randomized, controlled trial. J Am Soc Nephrol 17: 2307-2314

\section{Cyclophosphamide versus azathioprine for proliferative lupus nephritis therapy}

There is currently no consensus as to whether cyclophosphamide is better than azathioprine, in combination with corticosteroids, for the treatment of proliferative lupus nephritis—although azathioprine has less gonadal toxicity.

In this open-label trial, 87 patients with proliferative lupus nephritis were randomly allocated to 2 years of treatment with either intravenous pulsed cyclophosphamide $\left(750 \mathrm{mg} / \mathrm{m}^{2}, 13\right.$ pulses $)$ plus oral prednisone, or to oral azathioprine ( $2 \mathrm{mg} / \mathrm{kg}$ daily) plus intravenous pulsed methylprednisolone $(3 \times 3$ pulses of $1 \mathrm{~g})$ and oral prednisone. The 2-year incidence of partial or complete renal remission was similar in both groups. After a median follow-up of 5.7 years, however, doubling of initial serum creatinine level occurred more frequently in azathioprine-treated than in cyclophosphamide-treated patients 